\title{
Treatment of Hemodialysis-Associated Adynamic Bone Disease with Teriparatide (PTH 1-34): A Pilot Study
}

\author{
Daniel Cejka $^{\mathrm{a}}$ Katharina Kodras ${ }^{\mathrm{a}}$ Till Bader $^{\mathrm{b}}$ Martin Haas ${ }^{\mathrm{a}}$ \\ a Department of Internal Medicine III, Division of Nephrology and Dialysis, and bepartment of Radiology, \\ Division of Osteoradiology, Medical University Vienna, Vienna, Austria
}

\section{Key Words}

Teriparatide $\cdot \mathrm{PTH}_{1-34} \cdot$ Adynamic bone disease $\cdot$ Dialysis $\cdot$ Renal osteodystrophy

\begin{abstract}
Background: Adynamic bone disease (ABD) is caused by a relative or absolute parathyroid hormone (PTH) deficiency. Teriparatide $\left(\mathrm{PTH}_{1-34}\right)$ is an osteoanabolic agent in clinical use. Here, it was hypothesized that treatment with teriparatide improves bone mineral density (BMD) of ABD patients. Patients and Methods: Seven hemodialysis patients with ABD and a median iPTH level of $22 \mathrm{pg} / \mathrm{ml}$ were evaluated in this open-label, prospective, 6-month observational pilotstudy. All patients received $20 \mu \mathrm{g}$ teriparatide/day subcutaneously. Serologic bone markers, BMD and coronary artery calcification (CAC) were measured at baseline and after 6 months. Results: Teriparatide therapy led to a significant increase in lumbar spine $\left(0.885 \pm 0.08\right.$ vs. $0.914 \pm 0.09 \mathrm{~g} / \mathrm{cm}^{2}$, $\mathrm{p}<0.02)$, but not femoral neck (0.666 \pm 0.170 vs. $0.710 \pm$ $0.189 \mathrm{~g} / \mathrm{cm}^{2}, \mathrm{p}=0.18$ ) BMD. Compared to pretreatment values, calculated monthly changes in BMD improved significantly in both the lumbar spine and femoral neck $(p<0.02)$. Changes in serologic markers of bone turnover and CAC scores were not statistically significant. Conclusion: Teriparatide therapy might improve low BMD in hemodialysis patients with ABD. Further clinical studies are needed to establish teriparatide as a therapeutic option for dialysis patients with $A B D$.

Copyright $\odot 2010$ S. Karger AG, Basel
\end{abstract}

\section{Introduction}

Renal osteodystrophy (ROD), which is present in virtually all patients with end-stage renal disease (ESRD), is routinely classified into four major types: high-turnover ROD (osteitis fibrosa), mixed uremic osteodystrophy, osteomalacia and adynamic bone disease (ABD) [1]. ABD is currently the most common finding in dialysis patients and estimated to be present in approximately $40-50 \%$ [2]. Although bone mineral density (BMD) is generally decreased in ROD, patients with ABD seem to be particularly prone to hip fractures, which in turn is associated with increased mortality [3].

A broad range of different factors influence bone turnover [for review, see 4], however, the main causes for $\mathrm{ABD}$ are either reduced parathyroid hormone (PTH) synthesis or downregulation of PTH receptors in osteoblasts $[5,6]$. Due to the decreased efficacy of PTH in chronic renal failure intact PTH (iPTH) serum levels 2-9 times the upper normal limit are recommended in these patients [7].

Besides decreased bone density patients with ABD often suffer from hypercalcemia or hyperphosphatemia due to a reduced uptake of calcium and phosphate in bone $[8,9]$. Hyperphosphatemia in turn induces transforma-

Research funding: Jubiläumsfond der Österreichischen Nationalbank, grant No. 10281.

\section{KARGER}

Fax +41613061234 E-Mail karger@karger.ch www.karger.com
Martin Haas, MD

Department of Internal Medicine III, Division of Nephrology and Dialysis

Medical University Vienna

Währinger Gürtel 18-20, AT-1090 Vienna (Austria)

Tel. +43 140400 7255, Fax +43 140400 4392, E-Mail martin.haas@meduniwien.ac.at 
Table 1. Patient characteristics at the time of recruitment

\begin{tabular}{lllllllll}
\hline Patient, No. & Sex & Age & Fx & KTX & Dialysis, years & Dietary supplements & PTX & PTH, pg/ml \\
\hline 1 & M & 61 & 0 & Yes & 4.5 & Calcium & Yes & 20.5 \\
2 & F & 65 & 0 & Yes & 3 & Calcium + calcitriol & Yes & 44.6 \\
3 & F & 62 & 0 & Yes & 4 & Calcitriol & Yes & 2.9 \\
4 & F & 65 & 0 & No & 4.5 & Calcium + calcitriol & Yes & 14.7 \\
5 & F & 56 & 0 & No & 3.75 & Calcitriol & No & 25 \\
6 & M & 52 & 0 & Yes & 5 & Calcium + calcitriol & Yes & 125 \\
7 & M & 57 & 0 & Yes & 2.5 & Calcium & Yes & 22 \\
\hline
\end{tabular}

$\mathrm{Fx}=$ Bone fractures; KTX = previous kidney transplantation; PTX = parathyroidectomy.

tion of vascular smooth muscle cells into osteoblast-like cells [10]. In the presence of hypercalcemia the transformed cells then actively deposit calcium and phosphate into the vascular wall. This process seems to be more prevalent in ABD since the extent of vascular calcification is greater in low turnover than in normal or high turnover bone diseases [11]. Intermittently administered $\mathrm{PTH}_{1-34}$ (teriparatide) is able to stimulate osteoblasts and improve BMD $[12,13]$. Increased bone turnover would be particularly effective in patients with decreased parathyroid function, and has indeed also been shown in patients with normal kidney function and hypoparathyroidism [14]. We therefore conducted this prospective, open-labeled study in hemodialysis patients with inadequately low iPTH levels and low BMD.

\section{Patients and Methods}

Dialysis patients with ABD, determined by either bone biopsy or iPTH $<100 \mathrm{pg} / \mathrm{ml}$, osteopenia ( $\mathrm{T}$ score $<1$ at the femoral neck and/or lumbar spine), and hypo- or normocalcemia were eligible for this open, prospective 6-month intervention study.

Serologic parameters (osteocalcin (OC), bone alkaline phosphatase, iPTH, c-telopeptide, calcitriol, $25(\mathrm{OH})$-vitamin $\mathrm{D}_{3}$, calcium and phosphate), BMD and coronary calcification scores were measured at baseline and after 6 months. Besides absolute $B M D$ values the monthly BMD change was obtained by dividing the difference between two measurements by the number of months between the measurements. The monthly change before teriparatide administration was calculated similarly by using previous BMDs. The number of measurements before initiation of the study ranged between 1 and 5 , and the pretreatment observation period between 6 and 126 months.

Intact PTH was measured as previously reported [14]. All patients but 1 had undergone total parathyroidectomy with autotransplantation of 1 graft (table 1).

Subcutaneous recombinant human $\mathrm{PTH}_{1-34}(20 \mu \mathrm{g}$ teriparatide; FORTEO $^{\circledR}$; purchased from Eli Lilly, Indianapolis, Ind.,
USA) was administered once daily for 6 months. To avoid the development of osteomalacia during teriparatide treatment calcium-based phosphate binders and/or calcitriol were administered to all patients (table 1). The dose of both medications remained unchanged throughout the study.

The study was approved by the institutional review board (No. 146/2004) and all patients gave written informed consent before study inclusion.

Measurement of BMD and Coronary Artery Calcification

BMD measurements were performed with dual-energy X-ray absorptiometry (DEXA) and a QDR-4500 scanner (Hologic, Waltham, Mass., USA), using the manufacturer's recommended standard procedures for the posteroanterior lumbar spine at L1L4, and for the proximal femur at the femoral neck, trochanter, intertrochanteric region, total region, and Ward's triangle. The projectional BMD values are given in grams per square centimeter, and the individual patients' results are expressed as $\mathrm{Z}$ and $\mathrm{T}$ scores. The diagnostic bone-mass threshold for defining osteopenia was a $\mathrm{T}$ score of $<-1$ and for osteoporosis a T score of $<-2.5$.

The extent of coronary artery calcification (CAC) was measured with a multi-detector computer tomography (Sensation 16; Siemens Medical Systems, Forchheim, Germany) as previously reported [15]. The calcium score was determined on a commercially available external workstation and software ('syngo Calcium Scoring', Volume Wizard, Siemens). According to the method of Agatston et al. [16], we defined regions of interest by vessel and slice with the threshold option for pixels of $>130$ Houndsfield units (HU) to measure the area and peak density of plaques. The total calcium score was then calculated as the sum of the individual lesion scores in all coronary arteries.

\section{Bone Biopsy and Analysis}

A transcortical bone biopsy obtained from the iliac crest as previously reported [17] was performed in 1 patient with a pretreatment iPTH level of $125 \mathrm{pg} / \mathrm{ml}$ at the beginning and end of the study. Oral tetracycline was administered 3 and 1 week before biopsy. The specimen was embedded in plastic undecalcified and cut into 5 - $\mu \mathrm{m}$-thick sections. After standard staining the following histomorphometric parameters were evaluated: trabecular bone volume (BV/TV), osteoid volume (OV/TV), osteoid surface (OS/BS), osteoblast surface (Ob.S/BS); osteoclast surface (Oc.S/BS), eroded surface (ES/BS) and bone formation rate. 

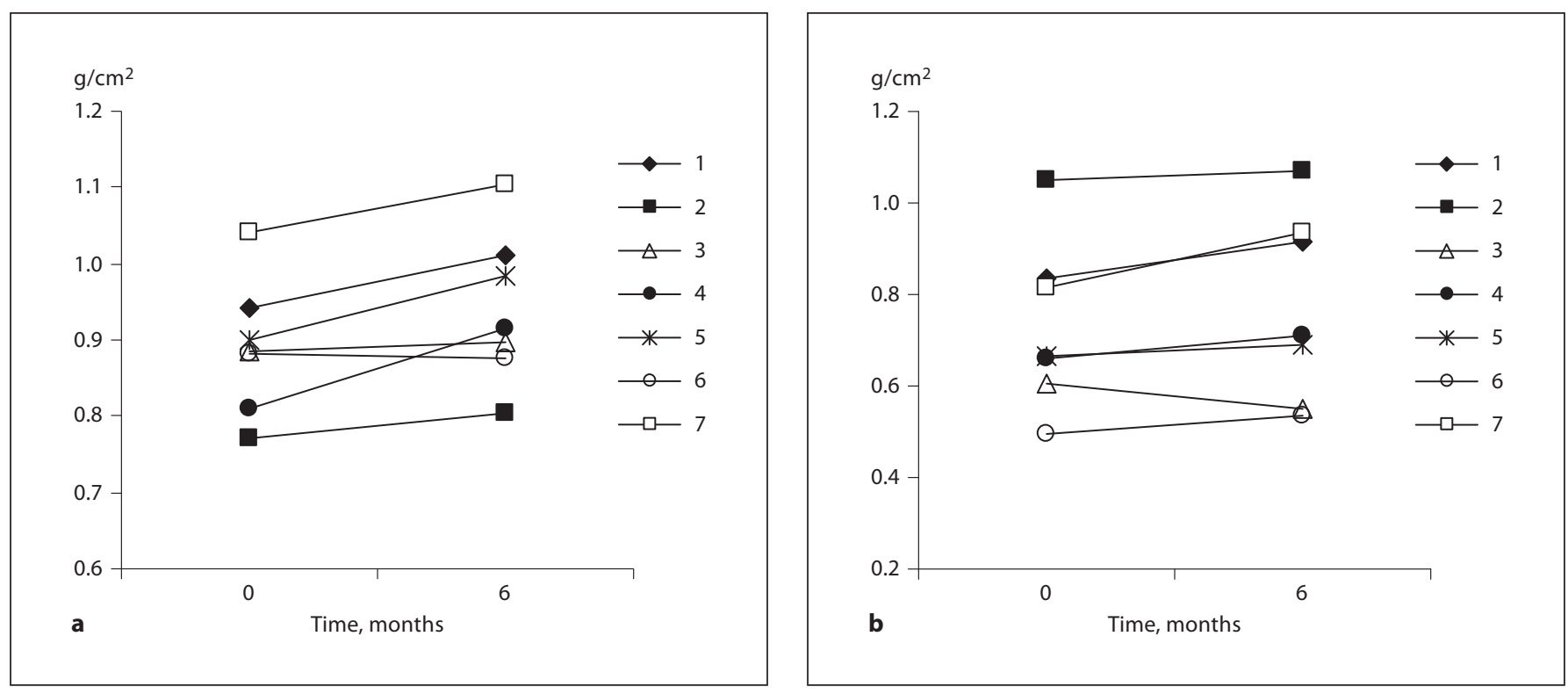

Fig. 1. Bone mineral density measured by dual X-ray absorptiometry (DEXA) of lumbar spine (a) and femoral neck (b) at baseline and after 6 months of therapy with teriparatide in individual patients.

\section{Statistics}

All results are expressed as median \pm SD. Differences between groups before and after therapy were tested for statistical significance by a two-tailed Wilcoxon test for matched pairs. Correlations between parameters were analyzed by the Spearman rank correlation test. $p$ values of $<0.05$ were considered statistically significant. Statistical analysis was performed using SPSS 12.0 software (SPSS Inc., Chicago, Ill., USA).

\section{Results}

Of 13 patients who were screened for the study, 3 patients were not eligible or were not interested in participating, thus 10 patients were enrolled. Three patients discontinued teriparatide therapy within 1 month after initiation of treatment because of lack of compliance $(n=1)$ or discomfort with the daily subcutaneous application $(n=2)$. A total of 7 patients were therefore included into the analysis. Six patients had had a previous parathyroidectomy with autotransplantation of 1 gland into the left arm. One of these patients (No. 6) had a PTH level of $>100 \mathrm{ng} / \mathrm{ml}$ (ng/l). However, a bone biopsy performed prior to inclusion confirmed ABD in this patient (no incorporation of tetracycline, normal osteoid and decreased bone volume). All other patients refused a bone biopsy. One patient (No. 2) received only 5 months of therapy with teriparatide because of renal transplantation. In this patient the serologic markers were obtained on the day of transplantation and bone density measurement within 1 week thereafter.

Patient characteristics are shown in table 1. Median age was $61 \pm 4.5$ years, and no participant had a known history of pathologic fracture. Time on hemodialysis at the time of study enrolment was $4.0 \pm 0.8$ years.

$\mathrm{BMD}$ of the lumbar spine increased significantly from $0.885 \pm 0.08$ to $0.914 \pm 0.09 \mathrm{~g} / \mathrm{cm}^{2}$ ( $\mathrm{p}<0.02$; fig. la) after 6 months of treatment with teriparatide. Measurements of the femoral neck showed a trend for increased BMD after 6 months of therapy from $0.666 \pm 0.170$ to $0.710 \pm 0.189 \mathrm{~g} / \mathrm{cm}^{2}$. However, this difference did not reach statistical significance ( $\mathrm{p}=0.18$; fig. $1 \mathrm{~b}$ ).

Teriparatide treatment led to a significant increase in monthly BMD rate in the lumbar spine as well as the femoral neck compared to long-term values. In the lumbar spine, the monthly change in BMD was $-0.035 \pm 0.20 \%$ before and $+0.965 \pm 0.653 \%$ after treatment with teriparatide $(\mathrm{p}<0.02)$. Correspondingly, the change in BMD of the femoral neck was converted from $-0.027 \pm 0.621$ to $1.317 \pm 1.169 \% /$ month $(\mathrm{p}<0.02)$.

Serum parameters of bone metabolism are shown in table 2. Serum phosphate showed a significant decrease from $5.52 \pm 0.75 \mathrm{mg} / \mathrm{dl}(1.78 \pm 0.24 \mathrm{mmol} / \mathrm{l})$ to $4.23 \pm$ $0.98 \mathrm{mg} / \mathrm{dl}(1.37 \pm 0.66 \mathrm{mmol} / \mathrm{l})$ after treatment with teriparatide compared to long-term pretreatment values 

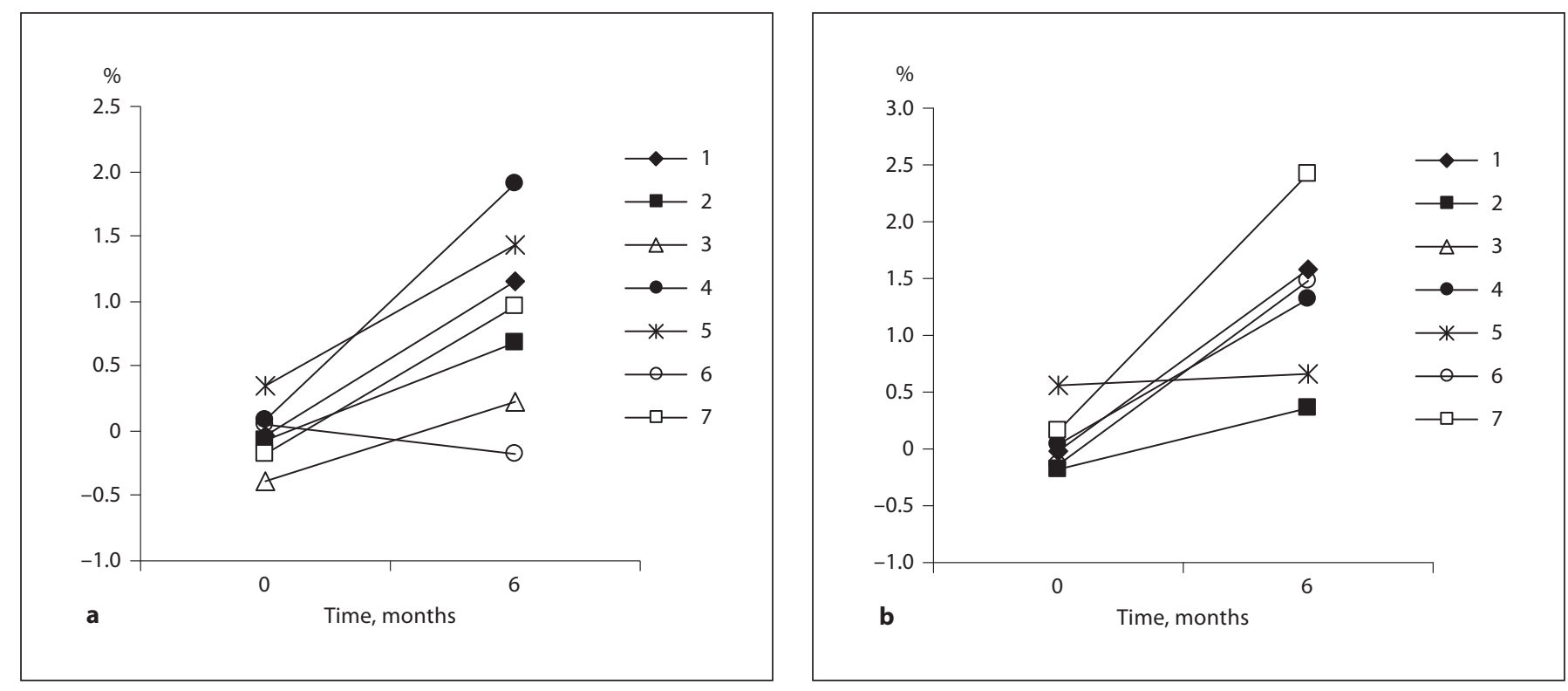

Fig. 2. Calculated percentage of monthly change in bone mineral density based on serial DEXA measurements of lumbar spine (a) and femoral neck (b) before and during therapy with teriparatide in individual patients.

Table 2. Serum parameters (median \pm SD) of patients before (baseline) and after 6 months (follow-up) of treatment with teriparatide

\begin{tabular}{lccl}
\hline & Baseline & Follow-up & p value \\
\hline Calcium, mg/dl & $8.80 \pm 0.67$ & $8.68 \pm 0.92$ & 0.61 \\
Phosphate, $\mathrm{mg} / \mathrm{dl}$ & $5.52 \pm 0.75$ & $4.23 \pm 0.98$ & 0.04 \\
$25-(\mathrm{OH})-\mathrm{D}_{3}, \mathrm{ng} / \mathrm{ml}$ & $22 \pm 16.4$ & $33 \pm 13.6$ & 0.60 \\
$1,25-(\mathrm{OH})_{2}-\mathrm{D}_{3}, \mathrm{pg} / \mathrm{ml}$ & $4.0 \pm 5.0$ & $15.0 \pm 7.7$ & 0.18 \\
$\mathrm{iPTH}, \mathrm{pg} / \mathrm{ml}$ & $22.0 \pm 38.0$ & $23.8 \pm 49.1$ & 0.39 \\
Osteocalcin, pg/ml & $146 \pm 126$ & $171 \pm 225$ & 0.13 \\
CTX, ng/ml & $1.60 \pm 0.88$ & $1.50 \pm 1.14$ & 0.31 \\
Bone AP, U/l & $20 \pm 31$ & $20 \pm 14$ & 0.49 \\
\hline
\end{tabular}

$25-(\mathrm{OH})-\mathrm{D}_{3}=25-$ Hydroxycholecalciferol; $1,25-(\mathrm{OH})_{2}-\mathrm{D} 3=$ 1,25-dihydroxycholecalciferol, calcitriol; iPTH = intact parathyroid hormone; CTX = type-I collagen peptides (cross-laps); bone $\mathrm{AP}=$ bone-specific alkaline phosphatase.

$(\mathrm{p}<0.04)$. Interestingly, the change in serum phosphate levels showed a negative correlation with the monthly increase in BMD of the femoral neck $(\mathrm{R}=-0.786, \mathrm{p}<0.03)$. All other changes in serum parameters were statistically not significant.

Paired data on CAC were available from 6 patients. Changes in CAC did not reach statistical significance. The median Agatston score before initiation of therapy was 361 (range 0-5,197) and 543 (range 17-5,254) after 6 months of teriparatide treatment $(\mathrm{p}>0.2)$. CAC increased in 4 patients and decreased in 3 patients. Changes in CAC were between -17 and $+272 \%$ compared to baseline values. No correlation was observed between changes in $\mathrm{BMD}$ and changes in CAC.

\section{Discussion}

The incidence of $\mathrm{ABD}$ has steadily increased during the last decades [17]. Mechanisms leading to ABD seem multifactorial. Importantly, chronic renal failure has been found to increase skeletal resistance to PTH by several mechanisms, such as truncated forms of PTH, downregulation of PTH receptors or increased levels of osteoprotegerin [14, 18]. In addition, total or subtotal parathyroidectomy, iatrogenic oversuppression of the parathyroid gland or low synthesis of PTH can lead to absolute PTH deficiency. Although the pathogenetic mechanisms are different, both forms of $\mathrm{ABD}$ have a reduced PTH effect on osteoblasts in common. This led to the recommendation of higher than normal PTH levels in chronic renal failure [7]. Therefore, we assumed that exogenous administration of PTH would abolish the relative or absolute PTH deficiency, and stimulate bone formation. 
In this study, treatment with $\mathrm{PTH}_{1-34}$ led to a significant increase in BMD of the lumbar spine in patients with ESRD. Moreover, based on serial BMD measurements, teriparatide converted a slow but continuous loss of BMD into a remarkable increase in BMD in the lumbar spine and femoral neck. These data suggest that teriparatide might be osteoanabolic in ESRD patients with serum iPTH below the recommended levels. This supports the hypothesis of relative PTH deficiency in ABD and the possibility to overcome this deficiency by exogenous administration of PTH. However, the iPTH levels were generally at the lower normal range. The cause for ABD in these patients is therefore rather PTH deficiency than PTH resistance. The marked effect on bone density by teriparatide may be less pronounced in patients with $\mathrm{ABD}$ and higher PTH levels.

A discrepancy to the increases in BMD is the absence of significant changes in serologic markers of bone turnover. Especially changes in OC, which increased only slightly, and bone-specific alkaline phosphatase, which remained unchanged during the course of the study would have been expected based on previous findings [19]. This might be explained by the imperfect correlation between serologic markers of bone turnover and bone turnover seen in bone biopsies from dialysis patients [20]. Another possibility is that statistical significance was not reached due to the small sample size and hence a type-II statistical error, especially in the case of OC.

However, there was a significant increase in the monthly change in BMD before and after teriparatide therapy. Considering that other therapies, especially calcium and vitamin D supplementation, remained unchanged, this change in bone mineralization dynamics is suggestive of an effect of teriparatide. Another possibility is that increases in aortic calcification may have contributed to increases in lumbar BMD, as influences of aortic calcification on lumbar BMD measurements have been reported to a variable extent $[21,22]$. Indeed, all patients included in this study had signs of calcification of the aorta on plain X-ray films. Since these calcifications were not quantified, data on progression of aortic calcifications are not available for analysis. In this study, the increase in BMD of the femoral neck of teriparatide-treated patients was weaker compared to increases in lumbar $\mathrm{BMD}$ and did not reach statistical significance. The lack of statistical significance might be explained by the small sample size. Furthermore, teriparatide exerts its bone anabolic effects predominantly on the axial skeleton [12, 19]. Thus, increases in femoral BMD might be expected to be lower than increases in BMD of the lumbar spine.
Teriparatide treatment was found to significantly decrease serum phosphate levels. Since elevated phosphate levels are positively correlated with increased mortality in ESRD [23], lowering of serum phosphate by teriparatide treatment might positively influence overall patient outcome. The change in serum phosphate was found to negatively correlate with an increase in BMD of the femoral neck. Thus, teriparatide might lead to increased utilization of serum phosphate in bone.

Prevention of cardiovascular disease is a major therapeutic goal, as ESRD is strongly associated with CAC even in young patients [24]. Decreasing serum phosphate levels have been suggested to protect arteries from pathologic calcium phosphate deposition [23]. In this study, teriparatide treatment was found to decrease serum phosphate levels, rendering amelioration of vascular calcification by teriparatide therapy possible. However, due to the high variation in vascular calcification among the subjects studied, conclusions on changes in CAC were severely hampered in this study. Interestingly, some patients experienced decreases in the Agatston score, while others showed marked increases in coronary calcification. Differences in susceptibility to vascular calcification in dialysis patients are well known and have been attributed to different levels of inhibitors of calcification [25]. Given this heterogeneity, further investigations in larger populations will therefore be necessary to assess the effect of teriparatide on vascular calcification.

Except for 1 patient who complained about bone pain and refused to continue treatment, no major side effects were observed. However, 2 patients found daily subcutaneous injections annoying and ended the study prematurely. This large dropout rate should be considered in future studies.

In conclusion, patients with ESRD and ABD showed increases in lumbar BMD after treatment with teriparatide. Recombinant PTH might have osteoanabolic effects in this subset of patients. Further studies are warranted to determine the impact of teriparatide treatment on bone metabolism, bone architecture and fracture incidence in patients with ESRD. 


\section{References}

$\checkmark 1$ Malluche H, Faugere MC: Renal bone disease 1990: an unmet challenge for the nephrologist. Kidney Int 1990;38:193-211.

$\checkmark 2$ Salusky IB, Goodman WG: Adynamic renal osteodystrophy: is there a problem? J Am Soc Nephrol 2001;12:1978-1985.

$\checkmark 3$ Coco M, Rush H: Increased incidence of hip fractures in dialysis patients with low serum parathyroid hormone. Am J Kidney Dis 2000;36:1115-1121.

$\checkmark 4$ Haas M: Renal osteodystrophy. Wien Med Wochenschr 2004;154:107-118.

5 Coen G: Adynamic bone disease: an update and overview. J Nephrol 2005;18:117-122.

-6 Iwasaki-Ishizuka Y, Yamato H, Nii-Kono T, Kurokawa K, Fukagawa M: Downregulation of parathyroid hormone receptor gene expression and osteoblastic dysfunction associated with skeletal resistance to parathyroid hormone in a rat model of renal failure with low turnover bone. Nephrol Dial Transplant 2005;20:1904-1911.

7 Kidney Disease: Improving Global Outcomes (KDIGO) CKD-MBD Work Group: KDIGO clinical practice guideline for the diagnosis, evaluation, prevention, and treatment of chronic kidney disease-mineral and bone disorder (CKD-MBD). Kidney Int Suppl 2009;113:S1-S130.

$\checkmark 8$ Kurz P, Monier-Faugere MC, Bognar B, Werner E, Roth P, Vlachojannis J, Malluche $\mathrm{HH}$ : Evidence for abnormal calcium homeostasis in patients with adynamic bone disease. Kidney Int 1994;46:855-861.

$\checkmark 9$ Fournier A, Yverneau PH, Hue P, Said S, Hamdini N, Eldin HM, Mohageb S, Oprisiu R, Marie A, Solal ME: Adynamic bone disease in patients with uremia. Curr Opin Nephrol Hypertens 1994;3:396-410.

$\checkmark 10$ Jono S, McKee MD, Murry CE, Shioi A, Nishizawa Y, Mori K, Morii H, Giachelli CM: Phosphate regulation of vascular smooth muscle cell calcification. Circ Res 2000; 87:E10-E17.
11 London GM, Marty C, Marchais SJ, Guerin AP, Metivier F, de Vernejoul MC: Arteria calcifications and bone histomorphometry in end-stage renal disease. J Am Soc Nephrol 2004;15:1943-1951.

12 Neer RM, Arnaud CD, Zanchetta JR, Prince R, Gaich GA, Reginster JY, Hodsman AB, Eriksen EF, Ish-Shalom S, Genant HK, Wang O, Mitlak BH, Mellstrom D, Oefjord ES, Marcinowska-Suchowierska E, Salmi J, Mulder H, Halse J, Sawicki AZ: Effect of parathyroid hormone (1-34) on fractures and bone mineral density in postmenopausal women with osteoporosis. N Engl J Med 2001;344: 1434-1441.

13 Prince R, Sipos A, Hossain A, Syversen U, Ish-Shalom S, Marcinowska E, Halse J, Lindsay R, Dalsky GP, Mitlak BH: Sustained nonvertebral fragility fracture risk reduction after discontinuation of teriparatide treatment. J Bone Miner Res 2005;20:1507-1513.

14 Haas M, Leko-Mohr Z, Roschger P, Kletzmayr J, Schwarz C, Domenig C, Zsontsich T, Klaushofer K, Delling G, Oberbauer R: Osteoprotegerin and parathyroid hormone as markers of high-turnover osteodystrophy and decreased bone mineralization in hemodialysis patients. Am J Kidney Dis 2002;39: 580-586.

15 Oschatz E, Benesch T, Kodras K, Hoffmann $\mathrm{U}$, Haas M: Changes of coronary calcification after kidney transplantation. Am J Kidney Dis 2006;48:307-313.

16 Agatston AS, Janowitz WR, Hildner FJ, Zusmer NR, Viamonte M Jr, Detrano R: Quantification of coronary artery calcium using ultrafast computed tomography. J Am Coll Cardiol 1990;15:827-832.

$\checkmark 17$ Couttenye MM, D’Haese PC, Verschoren WJ, Behets GJ, Schrooten I, De Broe ME: Low bone turnover in patients with renal failure. Kidney Int Suppl 1999;73:S70-S76.
18 Kazama JJ, Shigematsu T, Yano K, Tsuda E, Miura M, Iwasaki Y, Kawaguchi Y, Gejyo F, Kurokawa K, Fukagawa M: Increased circulating levels of osteoclastogenesis inhibitory factor (osteoprotegerin) in patients with chronic renal failure. Am J Kidney Dis 2002; 39:525-532.

19 Orwoll ES, Scheele WH, Paul S, Adami S, Syversen U, ez-Perez A, Kaufman JM, Clancy AD, Gaich GA: The effect of teriparatide [human parathyroid hormone (1-34)] therapy on bone density in men with osteoporosis. J Bone Miner Res 2003;18:9-17.

20 Bervoets A, Spasovski G, Behets G, Dams G, Polenakovic M, Zafirovska K, Van Hoof V, De Broe M, D’Haese P: Useful biochemical markers for diagnosing renal osteodystrophy in predialysis end-stage renal failure patients. Am J Kidney Dis 2003;41:997-1007.

21 Reid IR, Evans MC, Ames R, Wattie DJ: The influence of osteophytes and aortic calcification on spinal mineral density in postmenopausal women. J Clin Endocrinol Metab 1991;72:1372-1374.

22 Smith JA, Vento JA, Spencer RP, Tendler BE: Aortic calcification contributing to bone densitometry measurement. J Clin Densitom 1999;2:181-183.

$>23$ Block GA, Hulbert-Shearon TE, Levin NW, Port FK: Association of serum phosphorus and calcium $\times$ phosphate product with mortality risk in chronic hemodialysis patients: a national study. Am J Kidney Dis 1998;31:607-617.

$>24$ Goodman WG, Goldin J, Kuizon BD, Yoon C, Gales B, Sider D, Wang Y, Chung J, Emerick A, Greaser L, Elashoff RM, Salusky IB: Coronary-artery calcification in young adults with end-stage renal disease who are undergoing dialysis. N Engl J Med 2000;342: 1478-1483.

25 Cozzolino M, Mazzaferro S, Pugliese F, Brancaccio D: Vascular calcification and uremia: what do we know? Am J Nephrol 2008;28:339-346. 